\title{
¿Qué se crea al fomentar el emprendimiento? Los principales impactos de la formación en este campo*
}

What is Created by Promoting Entrepreneurship? The Main Impacts on Training in this Field

$\bigcirc$ que se cria ao fomentar o empreendimento? Os principais impactos da formação neste campo

\author{
Julián Cárdenas ${ }^{\star \star}$ \\ Alejandro Guzmán ${ }^{* *}$ \\ Carlos Sánchez ${ }^{\star \star \star \star}$ \\ Juan David Vanegas ${ }^{\star \star \star \star \star}$
}

Fecha de recibido: 21 de febrero de 2014

Fecha de aprobado: 29 de agosto de 2014

Doi: dx.doi.org/10.12804/rev.univ.empresa.28.2015.08

Para citar este artículo: Cárdenas, J., Guzmán, A., Sánchez, C., \& Vanegas, J. D. (2015). ¿Qué se crea al fomentar el emprendimiento? Los principales impactos de la formación en este campo. Universidad \& Empresa, 17(28), 173-190. Doi: dx.doi.org/10.12804/rev.univ.empresa.28.2015.08

\footnotetext{
Investigación financiada por el Parque del Emprendimiento de Medellín y la Universidad de Antioquia.

** Doctor en Sociología por la Universidad de Barcelona (España). Investigador en Freie Universität Berlin (Alemania) y profesor del Departamento de Sociología de la Universidad de Antioquia (Colombia). Web personal: www.networksprovidehappiness.com Correo electrónico: julian.cardenash@gmail.com. Dirección postal: Boltzmannstr. 1, 14195 Berlín, Alemania

*** Sociólogo de la Universidad de Antioquia. Profesor de la Universidad de Antioquia. Correo electrónico: ralejoguz@gmail.com

***** Sociólogo de la Universidad de Antioquia. Profesor de la Universidad de Antioquia. Correo electrónico: carlosdavid_7777@hotmail.com

****** Sociólogo de la Universidad de Antioquia. Docente Fundación Universidad Claretiana y en la Fundación Oscarez. Correo electrónico: juandavidvanegasdaza@hotmail.com
} 


\section{RESUMEN}

Las instituciones destinadas al fomento de la cultura emprendedora han crecido en número durante las últimas décadas. Estudios previos han mostrado la relación entre el fomento al emprendimiento y el crecimiento económico. Sin embargo, se desconocen los impactos políticos, culturales y sociales de dicha actividad. El objetivo de esta investigación es indagar si ese fomento impacta sobre el capital económico, humano y social, la solidaridad y la felicidad de las personas. Se encuestó a una muestra de individuos que se formaron en el Parque del Emprendimiento de Medellín (Colombia). Los resultados evidencian que el fomento del emprendimiento creó ciudadanos políticamente activos, aumentó su capital humano, impactó levemente en su capital económico y social, pero no creó sujetos más solidarios ni más felices. Palabras clave: capital humano, capital político, capital social, emprendimiento, felicidad.

\section{ABSTRACT}

During recent decades, there has been growth in the number of institutions whose purpose is to promote a culture of entrepreneurship. Previous studies have shown the relationship between the promotion of entrepreneurship and economic growth. However, the political, cultural and social impacts of this activity are unknown. The aim of this research is to ascertain whether such promotion affects economic, human and social capital and the solidarity and happiness of persons. A survey to a sample of individuals who were trained in the Medellin Entrepreneurship Park (Colombia) was applied. The results show that the promotion of entrepreneurship created politically active citizens, increased their human capital and slightly influenced on their economic and social capital. However, it did not create people who were either more collaborative or happier.

Keywords: Human capital, political capital, social capital, entrepreneurship, happiness.

\section{RESUMO}

As instituições destinadas ao fomento da cultura empreendedora têm crescido em número durante as últimas décadas. Estudos prévios têm mostrado a relação entre o fomento ao empreendimento e o crescimento económico. No entanto, desconhecem-se os impactos políticos, culturais e sociais de dita atividade. O objetivo desta pesquisa é indagar se esse fomento impacta sobre o capital económico, humano e social, a solidariedade e a felicidade das pessoas. Questionou-se a uma amostra de indivíduos que se formaram no Parque do Empreendimento de Medellín (Colômbia). Os resultados evidenciam que o fomento do empreendimento criou cidadãos politicamente ativos, aumentou o seu capital humano, impactou levemente no seu capital económico e social, mas não criou sujeitos mais solidários nem mais felizes.

Palavras-chave: capital humano, capital político, capital social, empreendimento, felicidade. 
INTRODUCCIÓN:

LOS IMPACTOS DEL

EMPRENDIMIENTO

El fenómeno del emprendimiento se ha estudiado desde hace más de un siglo. Se ha discutido su marco conceptual (Shane \& Venkataraman, 2000) y se han valorado los perfiles de los actores emprendedores (Delmar \& Davidsson, 2000; Solomon $\&$ Winslow, 1988), los determinantes de la actividad emprendedora (Evans \& Leighton, 1990; Reynolds, 1997) y, en menor medida, sus consecuencias o prevalencia laboral (Reynolds, Bygrave, Autio, Cox, \& Hay, 2002). Las investigaciones se han centrado en identificar personas con alta potencialidad emprendedora (descripción) y valorar qué las lleva a emprender (determinantes). Sin embargo, poco se conoce sobre qué genera el fomento del emprendimiento más allá del empleo (impactos).

La evidencia empírica sobre las consecuencias o impactos de los emprendedores ha estado centrada en los resultados económicos. Quienes miden el impacto económico de la actividad emprendedora, usando a las empresas como sujetos de análisis, han empleado como indicador de resultados económicos la supervivencia de la compañía (años de la organización) y su crecimiento empresarial (tamaño de la misma)
(Audretsch, Keilbach, \& Lehmann 2006; Caves, 1998).

Al relacionar el emprendimiento con el crecimiento económico de una región o un país (Audretsch \& Thurik, 2001; Reynolds, Brenda, \& Maki, 1995; Reynolds, Story, \& Westhead, 1994) los estudios han usado las medidas del Producto Interior Bruto (PIB) y la tasa de desempleo para calibrar qué ha generado el emprendimiento. Sin embargo, el fomento al emprendimiento va más allá del sector económico y laboral. Este puede tener además impactos políticos, humanos y sociales que requieren ser medidos para indagar qué ha generado en realidad este tipo de iniciativas.

En la actualidad, el fomento al emprendimiento está en auge. Desde el Estado se han diseñado e implementado políticas para crear una cultura emprendedora, financiar la creación de empresas y asesorar a emprendedores. Se ha asociado también al emprendimiento con las ideas de progreso, se han buscado estrategias para solucionar problemas de desempleo y pobreza y para promover la transferencia de conocimiento de la universidad al sector empresarial. En las últimas décadas, además, ha crecido el número de instituciones destinadas al fomento del espíritu emprendedor, así como la asesoría a emprendedores y la incubación de 
empresas. Centros como Barcelona Activa, Madrid Emprende en España o el Parque del Emprendimiento en Medellín, entre otros, han desarrollado durante décadas diversas iniciativas de fomento al emprendimiento.

El crecimiento de los programas educativos de emprendimiento y el aumento de recursos destinados al fomento de esta actividad ha generado un interés por parte de los proveedores, políticos y académicos por evaluar las consecuencias de formar emprendedores. Se han explorado los impactos directos (creación de empleo y nuevas iniciativas)e indirectos (aumento del espíritu emprendedor) de los programas de educación en esta materia (Bechard \& Toulouse 1998; Henry, Hill, \& Leitch, 2003). Otros estudios han señalado la relevancia de los programas educativos de emprendimiento en el aumento del capital humano, el desarrollo rural y la mejora en la gestión y dirección de organizaciones (Fayolle, Gailly, \& Lassas Clerc, 2006). Estas investigaciones han subrayado la complejidad del tema debido a la difícil visibilidad de estos efectos y la evaluación a largo plazo de la formación.

Indicar que el fomento al emprendimiento ayuda a crear empresas es limitado y, en buena medida, puede resultar algo obvio. La formación en valores de emprendimiento genera consecuencias que van más allá del empresarismo y de la mera creación de negocios. Existen amplios espacios que no han sido abordados aún en profundidad, como la relación entre emprendimiento y la creación de capital social y entre emprendimiento y la formación de ciudadanos políticamente activos o si el fomento de la cultura emprendedora genera personas más felices o más solidarias. Todas estas incógnitas e hipótesis de trabajo hacen interesante plantear una investigación sobre los impactos del fomento al emprendimiento en estos aspectos. De esta manera, puede ser posible disponer de una fotografía empírica que muestre para qué sirve y qué genera el fomento al emprendimiento.

Para alcanzar este objetivo se investigó el fenómeno considerando las experiencias vividas en el contexto específico del Parque del Emprendimiento de la ciudad de Medellín (Colombia). Nos centramos en analizar esto, porque allí se focalizan los esfuerzos, justamente, alrededor del fomento a la cultura emprendedora y no solo en realizar asesorías o incubación de empresas. Esto se hace mediante formaciones (capacitaciones) 
y eventos que buscan promover valores vinculados al emprendimiento. El Parque del Emprendimiento (Parque E) surge en 2006, con el impulso de la Alcaldía de Medellín y la Universidad de Antioquia. Sus líneas de acción se dirigen a la generación de cultura emprendedora y el apoyo y fortalecimiento de empresas. Estos propósitos se han ejecutado mediante actividades de sensibilización, formación, promoción y desarrollo, preincubación e incubación.

El fortalecimiento a la cultura emprendedora ha creado una nueva realidad social que va más allá de la mera creación de negocios. Analizando qué han hecho en materia de política, cultura, relaciones sociales, solidaridad y felicidad las personas que han sido formadas o capacitadas en este centro, es posible valorar e identificar qué impactos ha generado el fomento al emprendimiento en la sociedad. ¿Ha aumentado su nivel cultural? ¿Se han creado ciudadanos políticos? ¿Ha aumentado el capital social de estas personas? ¿Son más solidarias? ¿Son más felices? Esta investigación analiza justamente los comportamientos económicos, políticos, sociales, solidarios y de felicidad de las personas que han recibido formación en emprendimiento para averiguar cuáles son los impactos del fomento al emprendimiento.

\section{LOS IMPACTOS DEL EMPRENDIMIENTO EN LAS FORMAS DE CAPITAL}

Para abordar los potenciales espacios donde el fomento al emprendimiento puede haber tenido algún impacto en las personas que recibieron formación en esta materia, usamos la teoría de las formas del capital propuestas por Bourdieu (1986) y Coleman (1988). Estos autores señalan que las personas tienen diversos tipos de capital, los cuales pueden ser aumentados o disminuidos. Estos son: económico, humano, político y social. La diversidad de formas del capital llama la atención acerca de que el de tipo económico no es el único eje de estratificación y consecución de beneficios. Existen otros aspectos como el conocimiento (capital humano) y los contactos (capital social) que son igual o más relevantes para estratificar a las personas y saber qué pueden lograr. Es importante para la movilidad social, el ascenso, la promoción y la obtención de recursos, no solo cuánto se gana o se gasta, sino también cuánto se sabe y a quién se conoce.

Tanto el conocimiento como las redes son activos o capitales útiles para la supervivencia y crecimiento de las personas. En nuestra investigación utilizamos esta teoría sobre 
las formas del capital para diseñar un instrumento teórico y metodológico que nos ayuda a saber qué capitales ha generado en las personas la formación o capacitación en emprendimiento. Esta estrategia nos permitió medir en qué espacios ha impactado el fomento al emprendimiento y qué capitales desarrollan las personas formadas.

Estudiamos seis potenciales espacios en donde el Parque del Emprendimiento pudo haber tenido algún impacto en las personas formadas, es decir, que haya generado un valor agregado a las personas más allá de los meros contenidos del curso, a saber: impacto en el capital económico, el capital humano, el capital político, el capital social, en la solidaridad e impacto en la felicidad.

\subsection{Impacto en el capital económico}

El capital económico comprende los ingresos, gastos, inversiones, la propiedad de bienes y todas las otras fuentes de entradas. Para Bourdieu (1986) el capital económico posee un peso preponderante y decisivo frente a los otros tipos de capital, pues es la posesión de este lo que decide el éxito de las luchas en todos sus campos, es decir, es la especie de capital mejor convertibley constituye labase para la obtención de los demás.
El emprendimiento impacta en un primer momento directamente en el sector económico, pues fomenta la creación de empresas, el empleo y la acumulación de riquezas (Audretsch \& Thurik, 2001; Audretsch, Keilbach, \& Lehmann, 2006; Caves, 1998; Reynolds, Brenda, \& Maki, 1995; Reynolds, Story, \& Westhead, 1994). Sin embargo, nos preguntamos si la formación en emprendimiento también provoca un crecimiento económico en las personas, es decir, si la asistencia a capacitaciones sobre emprendimiento lleva a un aumento del capital económico medido a través de ingresos, gastos e inversiones.

\subsection{Impacto en el capital humano}

El capital humano es el conjunto de habilidades, conocimientos y aprendizajes de las personas que generan valor a nivel individual y social (Coleman, 1988). Está presente en la preparación académica, el aprendizaje y el conocimiento que poseen los individuos para desempeñarse en cualquier ámbito o empresa. Las personas más preparadas académicamente suelen tener mayores posibilidades de lograr el éxito en sus acciones y de ser más competitivas. Este tipo de capital está presente en el carácter de los seres humanos, que, por medio de sus habilidades y conocimientos, aumentan las posibilidades de producción (Sen, 2004). 
La mejora del capital humano trae consecuencias favorables a las sociedades ya que constituye la principal riqueza de una nación (Brunner \& Elacqua, 2003). No se trata solo de mejorar la base económica de las sociedades ni de buscar la modernización tecnológica de las mismas, se pretende un desarrollo del capital humano por las sinergias positivas que genera una sociedad del conocimiento y los profesionales. Los países con mayoresíndices de producción y distribución de conocimiento, así como con mayores índices educativos, tales como Noruega, Suecia y Canadá, son naciones con alto desarrollo económico, con menores índices de delincuencia, con una elevada calidad de vida y con un crecimiento sostenido a largo plazo (World Bank, 2007).

Una de las voluntades del fomento de la cultura emprendedora es la de estimular el crecimiento intelectual y el capital humano de las personas para afrontar de manera adecuada sus retos y contribuir al desarrollo social. Más allá de las competencias emprendedoras, el emprendimientodespierta un interés por el conocimiento y la formación continua. Para medir el desarrollo de capital humano, tras recibir formación en emprendimiento, se indaga acerca de si los actores han empezado estudios académicos, si tienen formación complementaria (capacitaciones), si han buscado más conocimiento sobre emprendimiento y más conocimiento general mediante lectura, la observación de noticias o la información acerca de la actualidad.

\subsection{Impacto en el capital político}

El campo político está intercalado ampliamente con el mundo socialy el económico (Bourdieu, 1986). Bourdieu hizo énfasis en la existencia de capital político porque esun activo intangible que puede ser utilizado para producir bienes y servicios (Viloria, Nevado, \& López, 2008, p. 17). Para este autor poseen capital político, en particular, los dirigentes, presidentes y reyes. Schugurensky (2000), por su parte, avanza en el concepto de capital político desplazando su exclusividad del terreno de los cargos gubernamentales a todos los individuos de la sociedad cuando estos tienen capacidades de liderazgo y representatividad. Por otro lado, Booth y Richard (1998) señalan que el capital político es la capacidad de influir en las decisiones políticas (para una revisión del concepto de capital político ver Nayden, 2011).

Usando las diversas concepciones, definimos capital político como la capacidad de influir en las decisiones de los demás. Se posee capital político en la medida en que se tiene 
fuerza para condicionar el entorno. De hecho, el liderazgo, el reconocimiento y el prestigio son cualidades decisivas para adquirir un mayor dominio del entorno y llevar a cabo iniciativas. No se trata de evaluar si las personas formadas en emprendimiento votan o hablan de política, sino de averiguar si la formación en emprendimiento genera personas líderes, populares y con fuerza para decidir sobre su entorno. Interesa conocer si la formación en emprendimiento forma actores que ejercen sus voluntades e influyen en su entorno cotidiano. Por esto analizamos el impacto en el capital político a partir de los indicadores de liderazgo, reconocimiento y prestigio. Si las personas son más líderes, más reconocidas y más prestigiosas tras la formación en emprendimiento, se podrá demostrar que mediante la promoción del emprendimiento se genera ciudadanos líderes y más activos en la sociedad.

\subsection{Impacto en el capital social}

En los años ochenta se erigió en las ciencias sociales el concepto de capital social para señalar que los contactos son un activo importante, es decir, que las redes son un capital. Académicos como Bourdieu (1986), Burt (1992), Coleman (1988), Lin (1999) y Putnam (1995) estudiaron las dimensiones del capital social.
Ellos destacaron la pertenencia a redes, la participación en organizaciones y la confianza como los elementos constitutivos.

Las redes (los contactos) constituyen un capital puesto que generan ventajas competitivas. Las personas bien conectadas, con redes amplias y heterogéneas, tienen más acceso a recursos, poder e información. Participar en organizaciones o asociaciones promueve relaciones sociales y permite movilizar recursos. Por otro lado, la confianza es constitutiva de capital social ya que sobre la base de la confianza en los demás se permiten mayores relaciones de reciprocidad. En los lugares en donde hay más confianza en la gente crecen las posibilidades de colaboración y el apoyo mutuo. En los espacios en donde hay alta desconfianza es más difícil emprender proyectos coordinados, con el consiguiente aumento de los costos de transacción y la caída de iniciativas conjuntas.

Desde múltiples instancias políticas y sociales se ha incentivado la construcción de relaciones que permitan construir redes sociales y que generen confianza entre los miembros de una comunidad o red social. El Banco Mundial ha trabajado sobre el capital social desde mediados de los años noventa. En su Informe sobre Desarrollo Mundial de 1997 (World 
Bank, 1997) esta entidad destaca la importancia que tiene el capital social para el crecimiento económico y el desarrollo, por cuanto las redes y la confianza favorecen el intercambio de información, la coordinación de actividades y la toma colectiva de decisiones. A partir de investigaciones realizadas alrededor del mundo por este banco y por la Organización para la Cooperación y el Desarrollo Económico (OCDE) se subraya que el capital social y la cohesión social son factores cruciales para aliviar la pobreza y lograr el desarrollo humano y económico sostenible (World Bank, 1999).

Aunque la relación entre redes, capital social y emprendimiento ha sido ampliamente analizada, exponiendo los tipos de redes empleadas por los emprendedores (Echeverri, 2009) y la necesidad de capital social para fomentar el emprendimiento (Burt, 2000; De Carolis \& Saparito, 2006; Davidsson \& Honig, 2003), poco se conoce sobre si formar una sociedad emprendedora favorece el desarrollo de capital social.

En la presente investigación analizamos si el fomento del emprendimiento supone un aumento del capital social medido a través de la creación de contactos, participación en asociaciones y confianza social. Descubrir que mediante la formación en emprendimiento se crea confianza en los demás, se aumenta la asociatividad y se crean redes sociales puede contribuir a rediseñar las políticas de cohesión social de empresas, comunidades y ciudades.

\subsection{Impacto en la solidaridad}

El auge del emprendimiento social y, en general, de iniciativas innovadoras destinadas principalmente a crear beneficios sociales (Mair, Battilana, \& Cardenas, 2012; Mair \& Martí, 2006) ha promovido la visión de que la formación en valores emprendedores genera mayor inquietud e incluso mayor solidaridad en las personas. Esto se debe a que la educación en competencias emprendedoras, como iniciativa, innovación y recursividad, permite a las personas desarrollar ideas y emprender proyectos ante realidades que no les gustan, así como llevar a cabo iniciativas que pueden ser beneficiosas para toda la sociedad. Se ha apoyado esta visión en países en vías de desarrollo y con un Estado del Bienestar mínimo, en donde el Estado no provee todos los servicios básicos a la población y se requieren iniciativas de beneficio para sectores en riesgo, es decir, proyectos sociales en donde ni el Estado ni el mercado llegan.

A pesar de lo anterior, este efecto solidario del fomento de las competen- 
cias emprendedoras ha sido ampliamente criticado por aquellos queconciben el emprendimiento como un camino a desarrollar valores comerciales y no sociales. Nuestra investigación pretende aportar a este debate evaluando el impacto del fomento de la cultura emprendedora en la solidaridad. Investigamos si tras la formación en emprendimiento se potenciaron acciones solidarias medidas a través de indicadores de ayuda a los demás, tolerancia y compromiso social.

\subsection{Impacto en la felicidad}

En los últimos años ha crecido el interés por estudiar y analizar la felicidad y la satisfacción personal y social. Entre el 2000 y el 2010 han aumentado exponencialmente los libros y artículos científicos que tratan de explicar la felicidad y la satisfacción (Rodríguez, Mohr, \& Arroyo, 2013). Se han destacado los factores sociales, económicos y educativos, más allá de los tradicionales de la salud, como determinantes de la felicidad. The New Economics Foundation ha desmitificado la idea del crecimiento económico y la felicidad a partir del uso y el análisis del Happy Planet Index. Este se basa en investigaciones cuantitativas realizadas en más de 170 países, en donde se pregunta a la gente por su felicidad, satisfacción y bienestar.
Nosotros nos preguntamos sobre el efecto del emprendimiento sobre la felicidad. Concretamente preguntamos si tras la formación recibida en emprendimiento los individuos eran más felices, se sentían mejor consigo mismos y si les gustaba más su trabajo. Aprender sobre emprendimiento puede traer apoyos más allá de los meros conocimientos empresariales, puede generar también relaciones y permitir aprender y conseguir un desarrollo cognitivo que pueda conducir al logro de una mayor felicidad. Descubrir que la formación en emprendimiento genera más felicidad podría suponer un cambio en las políticas de talento humano dentro de las empresas y en las políticas públicas, ya que mediante el fomento al emprendimiento se conseguiría alcanzar empleados y ciudadanos más felices.

\section{METODOLOGÍA Y DATOS}

Diseñamos un cuestionario de preguntas cerradas con el fin de averiguar los comportamientos llevados a cabo tras recibir cursos de formación en emprendimiento y que corresponden a los diferentes indicadores de capital humano, económico, político, solidaridad, felicidad y capital social. Preguntamos un conjunto de 76 ítems, mediante escala Likert de cinco puntos, donde los encuestados debían responder si estaban (5) 'totalmente de acuerdo', (4) 'de acuerdo', 
(3) 'ni de acuerdo ni en desacuerdo', (2) 'en desacuerdo' o (1) 'totalmente en desacuerdo'. Empleamos esta escala en lugar de preguntas dicotómicas (de Sí-No) para recoger con un mayor matiz y minuciosidad los diversos aspectos. El cuestionario fue validado por dos académicos que han trabajado en emprendimiento y por cinco profesionales que trabajan en instituciones dedicadas a este tema. Se realizó además una prueba piloto, con doce personas de la población de estudio.

Se construyeron índices de base 100 que permitieron comparar cuáles son los espacios en donde hay más impacto y aquellos en donde la incidencia es menor. Los índices de base 100 son escalas que oscilan entre 0 y 100 . La construcción de este índice se llevó a cabo mediante la técnica de índice sumatorio, ya que todas las preguntas fueron realizadas mediante escala Likert de 1 a 5. Tras la suma de las preguntas correspondientes a cada dimensión, se estandarizaron las puntuaciones para que escalaran entre 0 y 100 y se calculó una media por cada indicador. Proximidad a 100 señala alto impacto en esa dimensión y cercanía 0 indica un impacto nulo.

En esta investigación partimos de un conjunto de bases de datos suministradas por el Parque del Emprendi- miento de Medellín, en donde tenían registradas las personas que habían recibido cursos de formación en emprendimiento entre 2008 y 2010 . El universo o población son 2451 personas que recibieron cursos de formación y de los cuales se disponen datos de contacto. Obtuvimos una tasa de respuesta de $17,6 \%$, por lo que obtuvimos una muestra de 322 personas (con un error muestral del 5,1\% y un nivel de confianza de 95\%), de las cuales 165 son mujeres $(51,4 \%)$ y 156 hombres $(48,5 \%)$.

La distribución de la población, según el grupo de edad, fue la siguiente: el $50,6 \%$ de las personas que respondieron tenía entre 18 y 33 años, el $21,7 \%$ entre 34 y 45 y el $21,6 \%$ más de 46 años de edad. Respecto al estado civil 197 de las personas $(61,4 \%)$ son solteras, $91 \quad(28,3 \%)$ están casadas y el $18,3 \%$ del resto de la muestra está compuesta por viudos, separados y personas que viven bajo unión libre.

\section{RESULTADOS}

Formar personas en emprendimiento crea ciudadanos con alto capital político. De hecho, se obtuvo una puntuación total de 51, una de las más altas de todas. Esto muestra que las personas formadas en emprendimiento adquirieron una alta capacidad de influencia y reconocimiento 
en su entorno social (tabla 1). El capital político es medido a partir de los indicadores de liderazgo, reconocimiento y prestigio. Optamos por juntar los tres indicadores en un solo índice debido a que todos se correlacionan altamente. Potenciar valores de iniciativa, reducción del miedo al riesgo y recursividad en la formación en emprendimiento tiene consecuencias políticas en términos de influencia y prestigio en su entorno, tales como la creación de líderes ciudadanos y el posible desarrollo de movimientos sociales.

Tabla 1. Puntuaciones medias sobre base $\mathbf{1 0 0}$ de los posibles impactos tras la formación en emprendimiento

\begin{tabular}{|c|c|c|}
\hline Impactos en & Indicadores & $\begin{array}{c}\text { Puntuación media } \\
\text { sobre base } 100\end{array}$ \\
\hline Capital político & Liderazgo, reconocimiento, prestigio & 51,41 \\
\hline \multirow{4}{*}{ Capital humano } & Conocimiento general (noticias, lectura) & 54,18 \\
\hline & Formación complementaria & 34,34 \\
\hline & Educación académica & 18,37 \\
\hline & Conocimiento sobre emprendimiento & 16,02 \\
\hline \multirow{3}{*}{ Capital económico } & Inversiones & 20,16 \\
\hline & Gastos & 11,99 \\
\hline & Ingresos & 5,09 \\
\hline \multirow{3}{*}{ Capital social } & Redes de contactos & 19,96 \\
\hline & Confianza & 12,82 \\
\hline & Asociatividad & 8,86 \\
\hline \multirow{3}{*}{ Solidaridad } & Ayuda a los demás & 26,15 \\
\hline & Tolerancia & 15,03 \\
\hline & Compromiso social & 10,87 \\
\hline Felicidad & Felicidad & 9,52 \\
\hline
\end{tabular}

Fuente: elaboración propia. 
Formarse en emprendimiento lleva a querer seguir aprendiendo y aumentando el capital humano. En los datos analizados en cuanto a estefactor se observa que las personas que recibieron formación en emprendimiento desearon seguir adquiriendo nuevos conocimientos, especialmente generales, así como informarse acerca de noticias y actualidad, continuarleyendo prensa y libros, seguir consultado páginas web y capacitándose mediante cursos de formación complementaria.

En cuanto a continuar estudiando grados o posgrados el impacto es bajo. Esto seguramente debido a que la mayor parte de los encuestados son personas que ya han realizado estudios de grado e incluso algunos cuentan con estudios de posgrado y dominan otro idioma. Por este motivo, no está entre sus planes inmediatos realizar estudios adicionales. Finalmente, el conocimiento en temas de emprendimiento se encuentra muy reducido, este no les originó el seguir ahondando en temas de emprendimiento, posiblemente por la formación completa recibida en lo relativo a esta materia.

Tras el proceso de formación en emprendimiento las personas no obtuvieron más ingresos, sino mayores gastos, y realizaron inversiones mo- deradas. El análisis del capital económico mostró una puntuación por debajo de los 20 puntos, en una escala de cero a cien, lo que sugiere que no se ha creado más riqueza económica tras potenciar el emprendimiento. Este hallazgo puede depender mucho de la población estudiada, aunque sí pone de manifiesto que la asociación entre emprendimiento y el aumento del capital económico no siempre sucede y no necesariamente potenciando el emprendimiento crecen las economías. Posiblemente, la formación en emprendimiento ayuda al autoempleo, pero no necesariamente al desarrollo económico y el aumento significativo de los ingresos.

La formación en emprendimiento aumenta levemente el capital social de las personas. Los índices de capital social proyectaron una puntuación menor a los 20 puntos, en una escala de cero a cien. Tras la formación se produce muy poca participación en asociaciones y no se genera un incremento de la confianza en los demás. El único impacto destacable se produce en la creación de contactos. Gran parte del éxito de las actividades emprendedoras se basa en la creación de redes de colaboración y confianza en el entorno, lo que supone una reducción de los costos de transacción asociados a la vigilancia, el control y la negociación. 
Potenciar la cultura emprendedora no conduce a aumentar la solidaridad. Los datos arrojados para los índices de solidaridad muestran que la formación en emprendimiento no genera alto impacto en esta materia. Los índices de compromiso, tolerancia y solidaridad social no sobrepasan los treinta puntos en una escala del cero al cien. El aspecto más desarrollado fue el aumento en la ayuda a los demás, tal vez por la búsqueda de relaciones de reciprocidad de los potenciales emprendedores. Muy pocas personas manifestaron un aumento de la tolerancia y el compromiso social. Esto seguramente debido a que se han tendido a formar a emprendedores mediante valores poco colectivistas y más asociados con el individuo. La imagen del emprendedor como una persona individual arriesgada y con iniciativa no potencia valores de solidaridad.

Promover el emprendimiento no crea personas más felices. Juntamos los tres indicadores - felicidad, satisfacción en la vida y satisfacción laboral- en un solo índice, ya que correlacionan altamente. El índice sobre felicidad arrojó una puntuación menor a diez puntos, lo cual muestra que la formación en emprendimiento no provocó un impacto considerable en la satisfacción laboral e individual ni en la felicidad de las personas.

\section{DISCUSIÓN Y CONCLUSIONES}

Esta investigación pretendía medir el impacto del fomento de la cultura emprendedora en diversos sectores de la sociedad concentrándose en las experiencias identificadas en el Parque del Emprendimiento de Medellín, que realiza formación en emprendimiento. Hemos comprobado, en este contexto, que el fomento del emprendimiento genera más capital político y más capital humano en las personas; sin embargo, el impacto es leve en cuanto al capital económico y social y es bajo en materia de solidaridad y felicidad.

Es interesante observar cómo a más cultura emprendedora, se crean ciudadanos políticos activos y aumenta el interés por continuar estudiando y formarse intelectualmente. Sin embargo, nos llama la atención el bajo impacto en la creación de redes y el capital social. Esperábamos que la socialización en centros de formación fortaleciera los contactos, aumentara el tamaño de las redes y potenciara la confianza entre los asistentes. El aumento de la confianza en los demás y la asociatividad podría beneficiar proyectos colectivos o de colaboración entre los emprendedores, así como un mayor uso de las redes de apoyo para crear iniciativas o mejorar las existentes. Este hecho invita 
poderosamente a reflexionar sobre el concepto y la idea del emprendimiento. Tradicionalmente se ha asociado al emprendedor con una persona activay dinamizadora, perono conun grupo de personas activas o con un equipo de trabajo dinámico.

Posiblemente se esté enfatizando en gran medida la asociación entre emprendedor e individuo, y no entre emprendedor y grupo o sociedad, lo cual explica por qué hay también un bajo impacto en la solidaridad. Tal vez concebir el emprendimiento de manera diferente, como un equipo de personas colaborando, ayudaría a aumentar el impacto en la solidaridad. En una línea parecida, se ha acostumbrado a describir el emprendimiento como una acción de alto sacrificio, estresante y poco gratificante, lo cual puede explicar que las personas que recibieron formación en emprendimiento no resultaron ser más felices ni satisfechas.

En sociedades latinoamericanas como la colombiana, donde se ha dado mucha relevancia a la formación en emprendimiento desde las instituciones estatales y educativas, un cambio en la conceptualización del emprendimiento más dirigida a la creación de beneficios sociales, y no solo creación de negocios, ayudaría a crear sociedades más solidarias, comprometidas, con más confianza en los demás y posiblemente más satisfechas y felices.

A la larga, sociedades más cohesionadas y felices, producen más, crecen más y viven mejor. La formación de emprendedores debería reconfigurarse empezando por el mismo concepto de emprendedor para dejar de lado la vieja figura del líder empresarial solitario y mostrar al equipo de trabajo que suele ser el que realmente crea e inicia empresas.

¿Para qué sirve una sociedad de emprendedores? Esta es una inquietud que surge al pensar en qué sucede cuando se forman personas en iniciativa, manejo de riesgo y recursividad. El fomento del emprendimiento ha respondido desde las instituciones políticas y económicas a desarrollar económicamente ciudades y países, a generar autoempleo y a superar etapas de crisis. Sin embargo, pensar en una sociedad de emprendedores va más allá de lo meramente económico y requiere avanzar para preguntarse, en realidad, ¿qué genera el fomento del emprendimiento?

Aunque los datos analizados en esta investigación corresponden a una serie de experiencias particulares, alrededor de un centro que promueve el emprendimiento y forma emprendedores $y$, en consecuencia, los resultados no son extrapolables a toda la 
formación en emprendimiento, este estudio contribuye a ofrecer un marco conceptual y metodológico sobre cómo evaluar y medir las consecuencias o impactos que genera el fomento del emprendimiento. Futuras investigaciones podrían ir encaminadas a ampliar la muestra y tratar de medir si los resultados obtenidos son particulares de nuestro caso de estudio o son parte de un fenómeno generalizable. Parece importante reconocer que la cultura emprendedora no debe pensarse como un bien per se, desconociendo qué consecuencias genera en realidad.

\section{REFERENCIAS}

Audretsch, D. B., Keilbach, M. C., \& Lehmann, E. E. (2006). Entrepreneurship and Economic Growth. Oxford: Oxford University Press.

Audretsch, D., \& Thurik, A. (2001).

What's new about the new economy? Sources of growth in the managed andentrepreneurialeconomies. Industrial and Corporate Change, 10(1), 267-315.

Bechard, P., \& Toulouse, J. (1998). Validation of a didactic model for the analysis of training objectives in entrepreneurship. Journal of Business Venturing, 13(4), 317-332.

Booth, J. A., \& Richard, P. (1998). Civil society, political capital, and democratization in Central America. Journal of Politics, 60, 780-800.

Bourdieu, P. (1986). The forms of capital. En J. E. Richardson (Ed.), Handbook of theory and research for the sociology of education (pp. 242-258). Westport: Greenwood Press.

Brunner, J. J., \& Elacqua, G. (2003). Informe capitalhumanoenChile. Santiago de Chile: La Araucan. Burt,R.(1992).Structuralholes: The social structure of competition. Cambridge: Cambridge University Press.

Burt, R. S. (2000). The network structure of social capital. Research in Organizational Behavior, 22, 345-423.

Caves, R. E. (1998). Industrial organization and new findings on the turnover and mobility of firms. Journal of Economic Literature, 36(4), 1947-1982.

Coleman, J. (1988). Social capital in the creation of human capital. American Journal of Sociology, 94, 95-120.

Davidsson, P., \& Honig, B. (2003). The role of social and human capital among nascent entrepreneurs. Journal of Business Venturing, 18(3), 301-331.

De Carolis, D. M., \& Saparito, P. (2006). Social capital, cognition, and entrepreneurial opportunities: A theoretical framework. 
Entrepreneurship Theory and Practice, 30(1), 41-56.

Delmar, F., \& Davidsson, P. (2000). Where do they come from? Prevalence and characteristics of nascent entrepreneurs. Entrepreneurship \& Regional Development, 12(1), 1-23.

Echeverri, H. H. (2009). Investigación sobre redes sociales y emprendimiento: revisión de la literatura y agenda futura. Innovar, 19(33), 19-33.

Evans, D. S., \& Leighton, L. S.(1990). Small business formation by unemployed and employed workers. Small Business Economics, 2(4), 319-330.

Fayolle, A., Gailly, B., \& Lassas Clerc, N. (2006). Assessing the impact of entrepreneurship education programs: A new methodology. Journal of European Industrial Training, 30(9), 701-720.

Henry, C., Hill, F., \& Leitch, C. (2003). Entrepreneurship education and training. Aldershot: Ashgate Publishing, Ltd.

Lin, N. (1999). Building a network theory of social capital. Connections, 22(1), 28-51.

Mair, J., \& Martí, I. (2006). Social entrepreneurship research: A source of explanation, prediction, and delight. Journal of World Business, 41(1), 36-44.

Mair, J., Battilana, J., \& Cárdenas, J. (2012). Organizing for socie- ty: A typology of social entrepreneuring models. Journal of Business Ethics, 111(3), 353-373.

Nayden, N. (2011). Political capital conceptualization:Reclaimingthe heart of democracy [SSRN Scholarly Paper]. Rochester: Social Science Research Network.

Putnam, R. (1995). Bowling alone: America's declining social capital. Journal of Democracy, 6, 65-78.

Reynolds, P. (1997). Who starts new firms? Small Business Economics, 9, 449-462.

Reynolds, P. D., Bygrave, W. D., Autio, E., Cox, L. W., \& Hay, M. (2002). Global entrepreneurship monitor. Kansas City: Kaufman Center.

Reynolds, P., Brenda, M., \& Maki, R. (1995). Explaining regional variation in business. Small Business Economics, 7, 389-407.

Reynolds, P., Story, J., \& Westhead, P. (1994). Cross national comparison of variation on the new firm formation rates. Regional Studies, 27, 443-456.

Rodríguez, J.A., Mohr, J., \& Arroyo, L. (2013). Decoding happiness. Ponencia presentada a la XXXIII Sunbelt Conference, Hamburgo, Alemania.

Schugurensky, D. (2000). Citizenship learning and democratic engagement: Political capital revisited. Ponencia presentada a la 41 st 
Annual Adult Education Research Conference, Vancouver, Canadá. Sen, A. (2004). Rationality and freedeom. Cambridge: Harvard University Press.

Shane,S.,\&Venkataraman,S.(2000). The promise of entrepreneurship as a field of research. Academy of ManagementReview, 25,217-236.

Solomon, G. T., \& Winslow, E. K. (1988). Toward a descriptive profile of the entrepreneur. The Journal of Creative Behavior, 22(3), 162-171.

Viloria, G., Nevado, D., \& López, V. (2008). Medición y valoración del Capital Intelectual. España: Fundación EOI

World Bank (1997). World development report 1997: The state in a changing world. Washington: World Bank Publications.

World Bank (1999). Social capital for development. Recuperado el 6 de octubre de 2013, de http:// www.worldbank.org/poverty/ scapital/index.htm.

World Bank (2007). Building knowledge economies: Advanced strategies for development. Washington: World Bank Publications. 